-Editorial•

\title{
Recent progress in understanding the mechanisms of pain and itch
}

\author{
Ru-Rong Ji* \\ Sensory Plasticity Laboratory, Pain Research Center, Department of Anesthesiology, Perioperative and Pain Medicine, Brigham \\ and Women's Hospital and Harvard Medical School, 75 Francis Street, Boston, MA 02115, USA
}

Chronic pain is a major health problem world-wide. According to a recent report in the New England Journal of Medicine $^{[1]}$, the prevalence of pain in the United States is striking: more than 116 million Americans have pain that persist for weeks to years. Chronic pain is characterized as inflammatory pain, cancer pain, and neuropathic pain, and can result from such conditions as arthritis, cancer, diabetes, low back injury, surgery, viral infection, spinal cord injury, and stroke. It is estimated that the total financial cost of this epidemic is $\$ 560-635$ billion per year in the USA. It is worthy of note that the annual USA expenditure on pain (including direct medical costs and lost wages) is higher than that on cancer, heart disease, and diabetes combined. Despite the enormous costs, the treatments covered by these expenditures can only partially alleviate Americans' suffering. Comparatively, pain management in developing countries is far less than sufficient ${ }^{[2]}$.

Itch or pruritus is distinct from pain: while pain elicits a withdrawal reflex, itch evokes a desire to scratch. Although scratching can transiently alleviate itch, itch-scratch cycles often cause skin damage that exacerbates the itching problem. Chronic itch is associated with diseases of the skin, liver, and kidney, as well as metabolic disorders (e.g. diabetes $)^{[3,4]}$. Notably, chronic itch is resistant to current antihistamine treatments ${ }^{[3,4]}$.

Given the prevalence of pain and itch problems and the rapid increase in the number of scientists and clinicians who are interested in studying them, this special issue provides a timely focus on recent progress in our understanding of the mechanisms of pain and itch. Ten

*Guest Editor of the Special Issue, Associate Editor of Neuroscience Bulletin

Tel: +1-617-7328852; Fax: +1-617-7302801

E-mail: rrji@zeus.bwh.harvard.edu laboratories from China and the USA have contributed 11 papers to this special issue: 5 reviews and 6 full research articles $^{[5-15]}$. This special issue aims to cover several areas of research in pain and itch, from peripheral to central mechanisms, and also from neuronal to glial mechanisms.

This special issue starts with Ma's review that discusses population coding in somatic sensation and the relationship between pain and itch. While pain suppresses itch in normal conditions, this suppression can be lost under pathological conditions, due to changes in the spinal cord inhibitory circuitry ${ }^{[5]}$.

Both pain and itch are detected by primary sensory neurons such as dorsal root ganglion (DRG) neurons. Although the receptors in DRG neurons that sense noxious stimuli are well characterized, little is known about those that detect itchy (pruritic) stimuli. The mini-review by McNeil and Dong discusses the peripheral mechanisms of itch by focusing on the ligands and their receptors that directly cause primary sensory neuron activation and initiate itch sensation ${ }^{[6]}$. In another review, Liu and Ji propose a role of Toll-like receptors (e.g., TLR7), expressed by primary sensory neurons, in itch modulation ${ }^{[7]}$. Furthermore, they demonstrate that oxidative stress induces itching behavior (scratching) in mice, and this can be suppressed by antioxidants $^{[8]}$.

It is generally believed that chronic pain results from neural plasticity in both the peripheral (peripheral sensitization) and central nervous systems (central sensitization). Especially, activation of transient receptor potential ion channels (TRPs) in primary sensory neurons is essential for the generation of peripheral sensitization and pathological pain, as supported by several lines of evidence presented in three articles. First, Dong et al. demonstrate that TRPV1 function in DRG neurons is 
enhanced by local inflammation or stimulation of the chemokine CXCL1 ${ }^{[9]}$. Second, Han and colleagues show that TRPV1 expression in DRG neurons is up-regulated after exposure to formaldehyde and in the context of bone cancer pain ${ }^{[10]}$. Third, Ding et al. reveal that a TRPC channel antagonist can alleviate inflammatory pain ${ }^{[11]}$. Furthermore, Liu and Ji present data showing that TRPA1 but not TRPV1 is required for oxidative stress-induced $i_{c h}{ }^{[8]}$. As well as peripheral sensitization, central sensitization is also covered in Tao's review, focusing on the inflammationinduced sensitization of spinal cord neurons, which is modulated by the trafficking of glutamatergic AMPA receptors ${ }^{[12]}$.

Although pain is processed in neuronal networks, recent advances have also revealed the important roles of glial cells such as microglia and astrocytes in the pathogenesis of chronic pain. Liu and Ji discuss the roles of Toll-like receptors (TLRs 2-4) in regulating the activation of microglia and astrocytes in the spinal cord, leading to central sensitization and pain facilitation ${ }^{[7]}$. Guo and coauthors further demonstrate that the chemokine CCL2 can modulate descending pain facilitation in the brainstem via neuron-glia interactions ${ }^{[13]}$.

Opioids, such as morphine, are the first-line treatment for severe pain. Although morphine is very inexpensive to manufacture, $80 \%$ of the world's population, including more than 5 million patients with terminal cancer, do not have adequate access to this analgesic ${ }^{[2]}$. It is also known that long-term exposure to morphine has adverse effects, such as addiction and antinociceptive tolerance. It is of vital importance to find new ways of improving the efficacy of opioid analgesia and, meanwhile, reduce the side-effects. The review by Zhang and Bao provides several lines of evidence from their own and other laboratories that support the anatomical and functional interactions of $\mu$-and $\delta$-opioid receptors in primary sensory neurons and their central terminals ${ }^{[14]}$. They also discuss the controversy in the field ${ }^{[14]}$. The functional interactions of the $\mu$-and $\delta$-opioid receptors are further supported in the paper from Chen et al., reporting that the disruption of phosphorylation of the $\delta$-opioid receptor at a specific site (Threonine 161) can attenuate morphine tolerance in the context of inflammatory pain ${ }^{[15]}$.
It is my best wish that the special issue presented here will provoke new studies that provide a better understanding of the mechanisms underlying pain and itch, eventually leading to more effective treatments of devastating chronic pain and itch conditions.

\section{References:}

[1] Pizzo PA, Clark NM. Alleviating suffering 101-pain relief in the United States. N Engl J Med 2012, 366: 197-199.

[2] Lamas D, Rosenbaum L. Painful inequities - palliative care in developing countries. N Engl J Med 2012, 366: 199-201.

[3] Ikoma A, Steinhoff M, Stander S, Yosipovitch G, Schmelz M. The neurobiology of itch. Nat Rev Neurosci 2006, 7: 535-547.

[4] Paus R, Schmelz M, Biro T, Steinhoff M. Frontiers in pruritus research: scratching the brain for more effective itch therapy. J Clin Invest 2006, 116: 1174-1186.

[5] Ma Q. Population coding of somatic sensations. Neurosci Bull 2012, 28(2): 91-99.

[6] McNeil B, Dong X. Peripheral mechanisms of itch. Neurosci Bull 2012, 28(2): 100-110.

[7] Liu T, Gao YJ, Ji RR. Emerging role of Toll-like receptors in the control of pain and itch. Neurosci Bull 2012, 28(2): 131-144.

[8] Liu T, Ji RR. Oxidative stress induces itch via activation of transient receptor potential subtype ankyrin 1 in mice. Neurosci Bull 2012, 28(2): 145-154.

[9] Dong F, Du YR, Xie W, Strong JA, He XJ, Zhang JM. Increased function of the TRPV1 channel in small sensory neurons after local inflammation or in vitro exposure to the pro-inflammatory cytokine GRO/KC. Neurosci Bull 2012, 28(2): 155-164.

[10] Han Y, Li Y, Xiao X, Liu J, Meng XL, Liu FY, Xing GG, Wan Y. Formaldehyde up-regulates TRPV1 through MAPK and PI3K signaling pathways in a rat model of bone cancer pain. Neurosci Bull 2012, 28(2): 165-172.

[11] Ding J, Zhang JR, Wang Y, Li CL, Lu D, Guan SM, Chen J. Effects of a non-selective TRPC channel blocker, SKF-96365, on melittin-induced spontaneous persistent nociception and inflammatory pain hypersensitivity. Neurosci Bull 2012, 28(2): 173-181.

[12] Tao YX. AMPA receptor trafficking in inflammation-induced dorsal horn central sensitization. Neurosci Bull 2012, 28(2): 111-120.

[13] Guo W, Wang H, Zou S, Dubner R, Ren K. Chemokine signaling involving chemokine (C-C motif) ligand 2 plays a role in descending pain facilitation. Neurosci Bull 2012, 28(2): 193-207.

[14] Zhang X, Bao L. Interaction and regulatory functions of $\mu$ - and $\delta$-opioid receptors in nociceptive afferent neurons. Neurosci Bull 2012, 28(2): 121-130.

[15] Chen HJ, Xie WY, Hu F, Zhang Y, Wang J, Wang Y. Disruption of $\delta$-opioid receptor phosphorylation at Threonine 161 attenuates morphine tolerance in rats with CFA-induced inflammatory hypersensitivity. Neurosci Bull 2012, 28(2): 182-192. 